\title{
Analysis of embedded options in individual pension schemes in Germany
}

\author{
Alexander Kling · Jochen Russ • Hato Schmeiser
}

Received: 23 February 2004 / Revised: 12 July 2005

(C) Springer Science + Business Media, LLC 2006

\begin{abstract}
Newly introduced government-subsidized pension products in Germany are required to contain a promise by the seller to provide a "money-back guarantee" at the end of the term. The client is also given the right to stop paying premiums at any time (paid-up option). In this case, the amount of all premiums paid must also be guaranteed by the seller at maturity, no matter when the client stopped paying the premiums. Previous analyses of guarantees in such government-subsidized pension products have ignored this additional option. Within a generalized Black/Scholes framework, we analyze the value of the paid-up option for different products, market scenarios, and client behavior. Our results indicate that the paid-up option significantly increases the value of the money-back guarantee. Furthermore, we find that reducing volatility by shifting the client's assets from stocks to bonds as maturity approaches is a suitable means of reducing the risk arising from the "pure" moneyback guarantee but much less effective in reducing the risk arising from the paid-up option.
\end{abstract}

Keywords Individual pension schemes $\cdot$ Money-back guarantee $\cdot$ Option pricing

JEL Classification $\mathrm{G} 13 \cdot \mathrm{G} 23 \cdot \mathrm{G} 28$

\footnotetext{
A. Kling $\cdot \mathbf{J}$. Russ

Institut für Finanz- und Aktuarwissenschaften, Ulm, Germany

e-mail: a.kling@ifa-ulm.de

J. Russ

e-mail: j.russ@ifa-ulm.de

H. Schmeiser $(\square)$

Universität St. Gallen, Switzerland

e-mail: hato.schmeiser@unisg.ch
} 


\section{Introduction}

Newly introduced government-subsidized pension products in Germany (so called Riester-Products) are required to contain a promise by the seller ${ }^{1}$ to provide a "moneyback guarantee" at the end of the term. Hence, at minimum, the premiums paid by the client have to be available at the end of the accumulation phase $(T)$. Furthermore, the client is given the right to stop premium payments at any time. In this case, the amount of all premiums paid also must be guaranteed by the seller in $T$, no matter when the client stopped paying the premiums. Under the law, clients may become party to more than one subsidized contract, as long as the sum of the premiums paid into all contracts within each year does not exceed certain limits. ${ }^{2}$ Thus it is possible for a client to stop paying premiums on one contract and immediately take out a new contract (by the same or another provider). However, the money-back guarantee does not apply any longer if the client terminates the contract before the end of the accumulation phase ( $T$ ) and takes the money out of the contract. In this situation, the provider of the government-subsidized pension product has to pay out only the market value of the assets which can be lower than the sum of the premiums paid into the contract.

In discussions about the money-back guarantee in German government-subsidized pension products, product providers have often argued that-especially due to the long-term nature of this type of contract-the value of the investment at the end of the accumulation phase will almost certainly exceed the sum of all premiums paid and, hence, no specific risk management measures to ensure the money-back guarantee are necessary. However, for subsidized pension products offered by mutual funds, Gründl et al. [2004] have shown that even in the case where the client pays all premiums until maturity and there is a constant volatility, this argument is generally not true. Furthermore, the authors show that the money-back guarantee-which can be characterized as a put option on the underlying assets-can be of substantial value and does not seem to be adequately protected by current regulations.

In this paper, we consider the additional risk that can result if the client chooses to stop premium payment at some time during the life of the contract, which we refer to as the client making the contract paid-up. We look only at the case of subsidized pension products offered by mutual funds. If a contract is made paid-up at a point when the asset value of the contract is particularly low, it is possible for the client to speculate against the provider. In financial terms, this means that the client can choose to change the original guarantee into a new kind of guarantee at any time the original guarantee into a new kind of guarantee. The original guarantee is a put option on the assets in the contract and the assets to be bought by future premiums. The strike of the put option is the sum of all premiums scheduled to be paid throughout the life of the contract. The new guarantee, however, is a put option on the assets currently in the pension plan. The strike of this new put option is the sum of all premiums paid so far. The term of both put options is the remaining time to maturity. The option to

\footnotetext{
${ }^{1}$ In Germany, subsidized pension products can be sold by universal banks, insurance companies, and mutual funds.

${ }^{2}$ Details of the legal requirements in Germany are specified in the following laws: Altersvermögensgesetz (AvmG) and the Gesetz über die Zertifizierung von Altersvorsorgeverträgen (AltZertG).
} 
exchange the original guarantee with the new guarantee is given to the client for free, although its fair value may be positive.

Looking at our numerical results under different scenarios, we doubt that mutual funds are truly aware of the value of the guarantee they have given to their clients for free. ${ }^{3}$ However, it is very important to know the value of the money-back guarantee so as to price the contract appropriately. In an arbitrage-free market without transaction costs, the value of the put option is the price of any risk management measure that ensures the money-back guarantee. Hence, the seller of such subsidized pension products must receive the value of the put option from the clients in order to be able to finance adequate risk management measurers.

Our paper is organized as follows: in Section 2, we describe the current situation in Germany, explain why new subsidized pension products have been introduced, and describe the main features of such products. In Section 3, we describe the model used to analyze the value of the options. We assume a generalized Black/Scholes framework, allowing for a deterministic but possibly time-dependent term structure of interest rates, and assuming that the underlying assets follow a geometric Brownian motion with a deterministic but time-dependent volatility. The time-dependent volatility allows us to analyze if and by how much the embedded risks can be reduced by shifting from stocks to bonds during the life of the contract.

In Section 4, we derive methods for pricing the embedded options. First, we assume that the client pays all premiums from the start of the contract until maturity (i.e., we replicate the approach by Gründl et al. [2004]). As mentioned above, the money-back guarantee can be characterized as a put option on the underlying asset. Hence we price this put option using standard risk-neutral valuation techniques. Since we consider only contracts with a regular premium payment, no closed-form solutions for the option price exist (cf., e.g., Lachance and Mitchell [2003]). Thus we derive our results using Monte Carlo methods.

Second, we analyze how much the value of the embedded option is increased if we also allow the client to make the contract paid-up. In this context we analyze three different cases of client behavior and compare them with the situation outlined above, where we assumed that the investor would keep up the contract until the end of the savings term. As a point of reference, we start with the case where the investor takes out a contract and immediately stops premium payment after having paid the first premium. He then takes out a new contract, pays exactly one premium on the new contract and then stops making payments, and so forth. Hence, we have a series of forward-start put options on the underlying asset. Following a result in Russ [1999], we derive a closed-form solution for the value of these options and calculate the option values under different scenarios. Since the value of the money-back guarantee reaches its maximum in this situation, this case reveals the best strategy from the client's point of view.

\footnotetext{
${ }^{3}$ Even though we believe that, generally, subsidized pension products offered by mutual funds are not priced appropriately (the embedded options are typically given away for free), we do not think that this will create a solvency problem for the mutual funds because subsidized pension products are-at least at present-only a small part of the business written by German mutual funds. Hence we believe that there is no "option to default" (which would reduce the value of the money-back guarantee) for the sellers.
} 
We then consider the case where the investor stops premium payment at most once. First, we assume that the investor-by mere chance-discovers the optimal date to stop premium payment. In reality, this is not a possible strategy because it would require knowing the future, which in mathematical terms means that the strategy would not be adapted to the corresponding filtration. Nevertheless, this analysis quantifies the worst-case risk for the provider and should be taken into account in calculating the appropriate price. For valuation, we return to the standard principles of risk-neutral evaluation and assume that the investor makes the optimal decision based only on the available information. Finding this optimal strategy for exercising Bermuda-style options in a Monte Carlo framework is quite tricky. We solve this optimal stopping problem by using a result given in Douady [2002], describe our resulting Monte Carlo algorithm, and calculate the option values under different scenarios.

In the third and final case, we again allow the premium payment to be stopped at most once. However, we make the additional assumption that the investor, after having exercised the option, takes out a new contract into which the premiums are then paid until maturity. The methods used for this case are the same as above.

In Section 5, we display and analyze the results calculated by applying the methods explained in Section 4. All calculations are performed for several different underlyings and market scenarios. This sensitivity analysis helps identifying which kind of product and which client behavior is most risky under which market scenario.

\section{Government-subsidized pension schemes in Germany}

Massive problems in the state pension system caused by social and demographic changes have made private retirement provisions increasingly important in Germany. To make investing in private pension schemes more attractive, various financial incentives (tax relief and direct subsidies to the premiums) have been granted by the German government since the beginning of 2002. In principle, individuals are free to choose any pension contract offered by private financial institutions. However, the mentioned financial incentives are available only if certain criteria are met. ${ }^{4}$ One of the most important of these criteria is the money-back guarantee, as described above. As a consumer protection measure, sellers of private pension schemes are required to disclose all fees included in the premiums. Another requirement is that, generally, the investor must be at least 60 years of age at the end of the accumulation phase, at which point the investment typically is transferred into a lifelong annuity.

Two important points need explanation before continuing with our analysis. First, there is no legally required minimum contract duration for this new class of subsidized pension schemes; however, in practice, no contracts are offered with an accumulation phase that is less than five years. Second, according to the law, ${ }^{5}$ it is strictly forbidden for the manager of a mutual fund to use the investment of one group of clients to finance the money-back guarantee of another group of investors.

\footnotetext{
${ }^{4}$ For details of the legal requirements in Germany, see the Gesetz über die Zertifizierung von Altersvorsorgeverträgen (AltZertG).

${ }^{5}$ For details, see Section 9 (3) KAGG (Gesetz über Kapitalanlagegesellschaften). 


\section{The model framework}

\subsection{Analysis at the contract level}

We assume that all premiums paid by the client are invested in the underlying assets of the contract. For the sake of simplicity, we ignore administration or acquisition charges. Furthermore, we assume that the term to maturity is $T$ years, $T \in N$, and that all premium payments are made annually. ${ }^{6}$ The value of the underlying asset at time $t$ is denoted by $S_{t}$. If a client takes out such a contract at $t=0$ and pays an amount $P$ each year, the value of the assets after $t$ years (i.e., immediately before paying the $(t+1)^{\mathrm{st}}$ premium $)$ is given by

$$
V_{t}^{-}=\sum_{\nu=0}^{t-1} P \frac{S_{t}}{S_{\nu}}
$$

At the end of the term (i.e., at $t=T$ ), the client has paid $T$ premiums (at $t=$ $0, \ldots, T-1)$ and the value of the assets in the contract is

$$
V_{T}=V_{T}^{-}=\sum_{\nu=0}^{T-1} P \frac{S_{T}}{S_{\nu}} .
$$

Since the provider has given an asset value guarantee, the payoff to the investor (i.e., the value of the contract in $T$ ) is

$$
L_{T}=\max \left\{V_{T} ; T P\right\}=V_{T}+\left[T P-V_{T}\right]^{+} .
$$

Thus, the money the client receives is the value of the underlying assets plus the payoff of a put option on this value with strike $T P$.

If the client chooses to make the contract paid-up at time $t=\tau$ (i.e., before paying the $(\tau+1)^{\text {st }}$ premium), the value of the assets at maturity is given by

$$
V_{T}^{* \tau}=V_{\tau}^{-} \cdot \frac{S_{T}}{S_{\tau}}=\sum_{\nu=0}^{\tau-1} P \frac{S_{T}}{S_{\nu}} .
$$

However, the value of the contract at maturity is given by

$$
L_{T}^{* \tau}=\max \left\{V_{T}^{* \tau} ; \tau P\right\}=V_{T}^{* \tau}+\left[\tau P-V_{T}^{* \tau}\right]^{+}
$$

Again, this is the sum of the value of the underlying assets plus a put option on this value with strike $\tau P$.

\footnotetext{
${ }^{6}$ This last restriction is imposed because Monte Carlo algorithms for monthly premium payments are too time-consuming. We calculated some scenarios for monthly premium payment and found that the results are very similar.
} 
However, if the client cancels at $t=\tau$ and takes the money out of the contract, the money-back guarantee is no longer applicable. In this case, the payoff to the client at $\tau$ is the value of the assets $V_{\tau}^{-}$.

\subsection{A model for the economy}

We assume a finite time horizon $T$ and a complete, frictionless, and continuous market. We assume the value $S_{t}$ to follow a geometric Brownian motion (cf., e.g., Hull [2003]):

$$
\frac{d S_{t}}{S_{t}}=\mu(t)+\sigma(t) d W_{t}
$$

Here, $W_{t}$ denotes a Wiener process on some probability space $(\Omega, \Sigma, P)$ with a filtration $F$, to which $W$ is adapted. Both $\mu$ and $\sigma$ are deterministic but can be time dependent. For given $S_{0}$, the solution of Equation (6) is given by

$$
S_{t}=S_{0} e^{\int_{0}^{t} \mu(s)-\frac{\sigma^{2}(s)}{2} d s+\int_{0}^{t} \sigma(s) d W_{s}}
$$

hence, we have

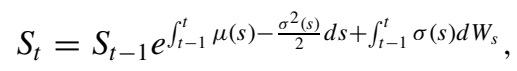

which is easily used in Monte Carlo algorithms.

The short rate process $r(t)$ is assumed to be deterministic and to fit the current, risk free term structure of interest rate, i.e.,

$$
\int_{t_{1}}^{t_{2}} r(t) d t=\left(t_{2}-t_{1}\right) f_{t_{1}, t_{2}}
$$

where $f_{t_{1}, t_{2}}$ denotes the continuous, annualized forward rate observed at time $t=0$ for the period of time $0 \leq t_{1}<t_{2}$. In our numerical examples, however, we let $r(t)=$ const.

In this setting, according to the Radon-Nikodym theorem, there exists a probability measure $Q$ so that the discounted price process $e^{-\int_{0}^{t} r(s) d s} S_{t}$ is a $Q$-martingale (cf., e.g., Harrison and Kreps [1979]). The price at time $t$ of any option considered here with payoff $O_{T}$ is then given by

$$
O_{t}=E_{Q}\left[e^{-\int_{t}^{T} r(s) d s} O_{T} \mid F_{t}\right], \quad 0 \leq t \leq T,
$$

where $E_{Q}\left[. \mid F_{t}\right]$ denotes the conditional expected value with respect to $Q$ under the information available at time $t .^{7}$ As a consequence of this transformation of measure, $\mu(t)$ is substituted by $r(t)$ in Equations (6), (7), and (8) and, hence, we have the

\footnotetext{
${ }^{7}$ Cf., e.g., Harrison and Pliska [1981] for details. 
risk-neutral process

$$
S_{t}^{*}=S_{0}^{*} e^{\int_{0}^{t} r(s)-\frac{\sigma^{2}(s)}{2} d s+\int_{0}^{t} \sigma(s) d W_{s}}
$$

or

$$
S_{t}^{*}=S_{t-1}^{*} e^{\int_{t-1}^{t} r(s)-\frac{\sigma^{2}(s)}{2} d s+\int_{t-1}^{t} \sigma(s) d W_{s}}
$$

respectively, which will be referred to by $S_{t}$ hereafter.

\section{Evaluation of the embedded options}

In this section we describe our methodology for pricing the embedded options under different scenarios. We derive formulas where possible, and explain the Monte Carlo algorithm where no closed-form solutions exist.

\subsection{Results without the paid-up option}

We start with the assumption that the client does not have the right to make the contract paid-up. In this case, the only embedded option is the asset value guarantee. This has been priced in Lachance and Mitchell [2003], as well as in Gründl et al. [2004]. For a constant volatility, constant interest rate, and monthly premium payments, our model and the model used in Gründl et al. [2004] coincide. The price at time $t$ of the embedded option is given by

$$
\Pi_{t}=E_{Q}\left[e^{-\int_{t}^{T} r(s) d s}\left[T P-V_{T}\right]^{+} \mid F_{t}\right]
$$

We calculate the price $\Pi:=\Pi_{0}$ by standard Monte Carlo methods using Equation (12) for generating paths of the underlying.

\subsection{Exercising the paid-up option annually}

We now assume that the investor takes out new contracts in yearly intervals and makes each contract paid-up after payment of the first premium. Thus, only one premium $P$ is paid into each contract and the guaranteed maturity value of each contract is $P$. In this case, the sum of the payoffs of the contracts at maturity is given by

$$
L^{(T)}=\sum_{\nu=0}^{T-1}\left(P \frac{S_{T}}{S_{v}}+\left[P-P \frac{S_{T}}{S_{v}}\right]^{+}\right)=V_{T}+\sum_{\nu=0}^{T-1}\left[P-P \frac{S_{T}}{S_{v}}\right]^{+} .
$$

Hence, the payoff is the value $V_{T}$ of the assets in the contracts plus the payoff of $T$ so-called forward-start put options with a payoff

$$
\left[P-P \frac{S_{T}}{S_{v}}\right]^{+}=\frac{P}{S_{v}}\left[S_{v}-S_{T}\right]^{+}, \quad v=0, \ldots, T-1 .
$$


The price at time 0 of such a forward-start performance option is denoted by $\Pi_{v}^{(T)}$ and given by

$$
\Pi_{\nu}^{(T)}=e^{-\int_{0}^{v} r(s) d s} \cdot P \cdot p_{v}(1,1, T-v)
$$

where $p_{t}(\alpha, \beta, \gamma)$ denotes the time $t$ value of a European put option on the underlying with spot price $\alpha$, strike $\beta$, and time to maturity $\gamma$. Thus, we have

$$
p_{t}(\alpha, \beta, \gamma)=\beta e^{-\int_{t}^{t+\gamma} r(s) d s} \Phi\left(-d_{2}\right)-\alpha \Phi\left(-d_{1}\right)
$$

with

$$
\begin{aligned}
d_{1} & =\frac{\ln \frac{\alpha}{\beta}+\int_{t}^{t+\gamma} r(s) d s+\frac{v^{2}}{2}}{v}, \\
d_{2} & =\frac{\ln \frac{\alpha}{\beta}+\int_{t}^{t+\gamma} r(s) d s-\frac{v^{2}}{2}}{v}, \\
v^{2} & =\int_{t}^{t+\gamma} \sigma^{2}(s) d s, \\
\Phi(x) & =\frac{1}{\sqrt{2 \pi}} \int_{-\infty}^{x} e^{-\frac{u^{2}}{2}} d u .
\end{aligned}
$$

A proof in the standard Black/Scholes framework follows from

$$
\begin{aligned}
\Pi_{v}^{(T)} & =E_{Q}\left[e^{-\int_{0}^{T} r(s) d s} \frac{P}{S_{v}}\left[S_{v}-S_{T}\right]^{+} \mid F_{0}\right] \\
& =E_{Q}\left[e^{-\int_{0}^{v} r(s) d s} E_{Q}\left[e^{-\int_{v}^{T} r(s) d s} \frac{P}{S_{v}}\left[S_{v}-S_{T}\right]^{+} \mid F_{v}\right] \mid F_{0}\right] \\
& =E_{Q}\left[e^{-\int_{0}^{v} r(s) d s} \frac{P}{S_{v}} p_{v}\left(S_{v}, S_{v}, T-v\right) \mid F_{0}\right] \\
& =E_{Q}\left[e^{-\int_{0}^{v} r(s) d s} \cdot P \cdot p_{v}(1,1, T-v) \mid F_{0}\right] \\
& =e^{-\int_{0}^{v} r(s) d s} \cdot P \cdot p_{v}(1,1, T-v) .
\end{aligned}
$$

The generalization for time-dependent volatility and short rate follows from a result in Russ [1999, Section 3.2.2] and the put-call parity.

With these results, the value of the embedded option is given by

$$
\Pi_{S}^{(T)}=\sum_{\nu=0}^{T-1} \Pi_{\nu}^{(T)}=\sum_{\nu=0}^{T-1} e^{-\int_{0}^{\nu} r(s) d s} \cdot P \cdot p_{\nu}(1,1, T-\nu)
$$


The German market offers products without up-front charges, i.e., all charges are included as a flat rate percentage of each premium. Thus a client following the strategy explained in this section would not pay higher charges than a client paying all premiums into one contract. Hence, this is the best strategy from the investor's point of view.

\subsection{Exercising the paid-up option once without entering a new contract}

In this section we consider the case where the investor exercises the paid-up option only once. Since the option is a Bermuda-style option, finding the optimal exercise date is not trivial. To analyze the worst case from the product provider's point of view, ${ }^{8}$ we first assume that the investor makes the contract paid-up at some point of time and by mere chance chooses the optimal date $\tau$ in the following sense:

$$
\left[\tau P-V_{T}^{* \tau}\right]^{+}=\max _{1 \leq t \leq T}\left\{\left[t P-V_{T}^{* t}\right]^{+}\right\}
$$

In this case we denote the price of the option by $\Pi_{K}^{(1)}$ (the index (1) indicates that only one contract is involved and $K$ indicates that we assume the client to know the future; where we assume the client to follow a more realistic strategy (see above), the index $K$ is substituted by $S$ ). The option price $\Pi_{K}^{(1)}$ is given by:

$$
\Pi_{K}^{(1)}=E_{Q}\left[\max _{1 \leq t \leq T}\left\{e^{-\int_{0}^{T} r(s) d s}\left[t P-V_{T}^{* t}\right]^{+}\right\} \mid F_{0}\right]
$$

This can be calculated by a simple Monte Carlo algorithm, where

$$
\pi_{K}^{(1)}=\frac{1}{\operatorname{sim}} \sum_{i=1}^{\operatorname{sim}} \max _{1 \leq t \leq T}\left\{e^{-\int_{0}^{T} r(s) d s}\left[t P-v_{T}^{(i) * t}\right]^{+}\right\}
$$

is used as a Monte Carlo estimate for the option price. Here, sim denotes the number of simulations and for each $i=1, \ldots$, sim, a path of the underlying is generated and thus the values $v_{T}^{(i) * t}$ are realizations of $V_{T}^{* \tau}$.

Finding the optimal exercise date for Bermuda-style options is an optimal stopping problem (cf., e.g., Anderson [1999]). The value $\Pi_{K}^{(1)}$ calculated above is not the price of the option but, rather an upper bound, as finding the exercise date would require knowledge of future events. Thus, the random variable $\tau$ that describes when to exercise the option is no stopping time and hence not an admissible strategy. Admissible strategies only use information known at the present time, i.e., $\tau$ has to be adapted to the filtration $F$. In Douady [2002], it is shown that whenever the underlying model for the economy has only one source of uncertainty, an optimal admissible strategy for exercising Bermuda-style options can be found by looking only at the exercise value of the option. In our case, this is apparently equivalent to considering only the current value of the underlying assets $V_{t}^{-}$.

\footnotetext{
${ }_{8}$ In general, this will be the worst case for the provider since most sellers of government-subsidized pension products allow their clients to use the paid-up option only once per contract. Hence it is not possible to pay more premiums into a contract that has previously been made paid-up.
} 
We call a subset $K=K_{1} \times \cdots \times K_{T-1} \subseteq R^{T-1}$ an exercise strategy. Following this strategy, the option is exercised at time $t$ if and only if the option has not been exercised before and $V_{t}^{-} \in K_{t}$, i.e.,

$$
1_{K_{t}}\left(V_{t}^{-}\right)=1 \quad \text { and } \quad 1_{K_{s}}\left(V_{s}^{-}\right)=0 \quad \forall s \in\{1, \ldots, t-1\}
$$

The value of the option under the assumption that the investor applies some given strategy $K$ is given by

$$
\Pi^{(1)}(K)=E_{Q}\left[e^{-\int_{0}^{T} r(s) d s}\left[\tau P-V_{T}^{* \tau}\right]^{+} \mid F_{0}\right]
$$

where

$$
\tau=\inf \left\{t \in\{1, \ldots, T-1\}: V_{t}^{-} \in K_{t}\right\}
$$

or $\tau=T$, if $V_{t}^{-} \notin K_{t} \forall t \in\{1, \ldots, T-1\}$. This value can be calculated by Monte Carlo methods using the algorithm given in Appendix A.

Douady [2002] and Anderson [1999] describe the so-called exercise value threshold method (EVTM) to approximate an optimal strategy, i.e., a strategy maximizing $\Pi^{(1)}(K)$ by Monte Carlo methods. They show the existence of an optimal strategy of the form exercise if and only if for the first time $A_{t} \geq c_{t}$, where

$$
A_{t}=E_{Q}\left[e^{-\int_{t}^{T} r(s) d s}\left[t P-V_{t}^{-} \frac{S_{T}}{S_{t}}\right]^{+} \mid F_{t}\right]
$$

and $c_{t} \in R \forall t$. In our setting this translates to a strategy $K=\left(0 ; k_{1}\right] \times \cdots \times\left(0 ; k_{T-1}\right]$ for some $k_{i} \in R, i=1, \ldots, T-1$. We use a backward induction algorithm given by Douady [2002] to determine the optimal values $k_{1}, \ldots, k_{T-1}$. This algorithm is adapted into our setting and used it to determine an optimal strategy. The value of the option using an optimal adapted strategy is denoted by $\Pi_{S}^{(1)}$. The resulting algorithm is given in Appendix B.

\subsection{Exercising the paid-up option once and entering a new contract}

We now look at the case where, after having exercised the paid-up option, the investor takes out a new contract into which the premiums are then paid until maturity. The value $V_{T}^{* t}$ of the assets and hence the value $L_{T}^{* t}$ of the first contract at maturity, if the client chooses to make the contract paid-up at time $t=\tau$, is given in Section 3.1. If the client then starts paying premiums for a second contract and continues the payments until maturity, the value $W_{T}^{* t}$ of the assets of this second contract is given by

$$
W_{T}^{* \tau}=\sum_{\nu=\tau}^{T-1} P \frac{S_{T}}{S_{\nu}}
$$


The value of the investor's portfolio at maturity is given by

$$
\begin{aligned}
\tilde{L}_{T}^{* \tau} & =\max \left\{V_{T}^{* \tau} ; \tau P\right\}+\max \left\{W_{T}^{* \tau} ;(T-\tau) P\right\} \\
& =\sum_{\nu=0}^{T-1} P \frac{S_{T}}{S_{\nu}}+\left[\tau P-V_{T}^{* \tau}\right]^{+}+\left[(T-\tau) P-W_{T}^{* \tau}\right]^{+} .
\end{aligned}
$$

This is the value of the underlying assets plus two put options.

Again, we first assume that the investor chooses the optimal point in time for exercising the paid-up option by chance in the following:

$$
\begin{aligned}
& {\left[\tau P-V_{T}^{* \tau}\right]^{+}+\left[(T-\tau) P-W_{T}^{* \tau}\right]^{+}} \\
& \quad=\max _{1 \leq t \leq T}\left\{\left[t P-V_{T}^{* t}\right]^{+}+\left[(T-t) P-W_{T}^{* t}\right]^{+}\right\} .
\end{aligned}
$$

In this case, the price of the option is given by

$$
\Pi_{K}^{(2)}=E_{Q}\left[\max _{1 \leq t \leq T}\left\{e^{-\int_{0}^{T} r(s) d s}\left(\left[t P-V_{T}^{* t}\right]^{+}+\left[(T-t) P-W_{T}^{* t}\right]^{+}\right)\right\} \mid F_{0}\right]
$$

and can be calculated by a simple Monte Carlo algorithm, where

$$
\pi_{K}^{(2)}=\frac{1}{\operatorname{sim}} \sum_{i=1}^{\operatorname{sim}} \max _{1 \leq t \leq T}\left\{e^{-\int_{0}^{T} r(s) d s}\left(\left[t P-v_{T}^{(i) * t}\right]^{+}+\left[(T-t) P-w_{T}^{(i) * t}\right]^{+}\right)\right\}
$$

is used as a Monte Carlo estimate for the option price. As before, sim denotes the number of simulations and for each $i=1, \ldots, \operatorname{sim}$ a path of the underlying is generated and thus the values $v_{T}^{(i) * t}$ and $w_{T}^{(i) * t}$ are realizations of $V_{T}^{* \tau}$ and $W_{T}^{* \tau}$.

Once again, $\tau$ is no stopping time. We thus define an exercise strategy as a subset $\tilde{K}=\tilde{K}_{1} \times \cdots \times \tilde{K}_{T-1} \subseteq R^{T-1}$ and exercise the paid-up option if and only if

$$
1_{\tilde{K}_{t}}\left(V_{t}^{-}\right)=1 \quad \text { and } \quad 1_{\tilde{K}_{s}}\left(V_{s}^{-}\right)=0 \quad \forall s \in\{1, \ldots, t-1\}
$$

Given any strategy $\tilde{K}$ the value of the option is

$$
\Pi^{(2)}(\tilde{K})=E_{Q}\left[e^{-\int_{0}^{T} r(s) d s}\left(\left[\tau P-V_{T}^{* \tau}\right]^{+}+\left[(T-\tau) P-W_{T}^{* \tau}\right]^{+}\right) \mid F_{0}\right],
$$

where

$$
\tau=\inf \left\{t \in\{1, \ldots, T-1\}: V_{t}^{-} \in \tilde{K}_{t}\right\}
$$

or $\tau=T$, if $V_{t}^{-} \notin \tilde{K}_{t} \forall t \in\{1, \ldots, T-1\}$. $\Pi^{(2)}(\tilde{K})$ can be calculated by Monte Carlo methods using the algorithm given in Appendix $\mathrm{C}$. 
Again we use EVTM to maximize $\Pi^{(2)}(\tilde{K})$ by Monte Carlo methods. This means we exercise the paid-up option if and only if for the first time $\tilde{A}_{t} \geq \tilde{c}_{t}$, where

$$
\tilde{A}_{t}=E_{Q}\left[e^{-\int_{t}^{T} r(s) d s}\left(\left[t P-V_{T}^{* t}\right]^{+}+\left[(T-t) P-W_{T}^{* t}\right]^{+}\right) \mid F_{t}\right]
$$

and $\tilde{c}_{t} \in R \forall t$. As shown above, this translates to a strategy $\tilde{K}=\left(0 ; \tilde{k}_{1}\right\rfloor \times \cdots \times$ $\left(0 ; \tilde{k}_{T-1}\right\rfloor$. Once more, we use a backward induction algorithm to determine the optimal values $\tilde{k}_{1}, \ldots, \tilde{k}_{T-1}$, which is given in Appendix $\mathrm{D}$. We denote the value of the option using an optimal adapted strategy by $\Pi_{S}^{(2)}$.

\section{Numerical results}

In this section we display and analyze some numerical results based on the methods developed in Section 4. We consider the following contracts, where regular annual premiums are paid over the whole term of the contract:

- Contract 1: Annual premium payments of 8,400€; term = 5 years.

- Contract 2: Annual premium payments of 4,200 €; term $=10$ years.

- Contract 3: Annual premium payments of 2,100 €; term $=20$ years.

- Contract 4: Annual premium payments of $1,200 €$; term $=35$ years.

For each of these contracts, the sum of the premiums paid at maturity is $42,000 €$ if the client has not previously made the contract paid-up.

As mentioned before, we assume that the client's premiums are invested in mutual funds. The funds are assumed to invest in stocks and bonds. There are products available where all clients invest in the same fund, independent of the remaining term to maturity. Such products are modelled with a constant volatility. The law allows such funds to have a stock portion of up to $100 \%$.

Numerous products are available in Germany redeploying the clients' assets to funds with a higher bond portion (and thus a lower volatility) as time to maturity decreases. Our model considers such products by assuming that the volatility of the underlying asset is decreasing. Table 1 gives an overview of the used underlyings' volatilities in our numerical examples.

Tables 2-5 show the option prices for the four contract forms and the six different underlyings, given a (constant) short rate of 5\%. Again, $\Pi$ stands for the price of the put option without the paid-up option, $\Pi_{S}^{(T)}$ denotes the case where the paid-up

Table 1 Volatility structure (in percent) of the underlyings used in the calculations

\begin{tabular}{llllllllll}
\hline Time to maturity in years & $35-21$ & $20-16$ & $15-11$ & $10-6$ & 5 & 4 & 3 & 2 & 1 \\
\hline Underlying 1 & 20 & 20 & 20 & 20 & 20 & 20 & 20 & 20 & 20 \\
Underlying 2 & 15 & 15 & 15 & 15 & 15 & 15 & 15 & 15 & 15 \\
Underlying 3 & 10 & 10 & 10 & 10 & 10 & 10 & 10 & 10 & 10 \\
Underlying 4 & 20 & 20 & 20 & 20 & 20 & 20 & 15 & 15 & 10 \\
Underlying 5 & 20 & 20 & 15 & 10 & 5 & 5 & 5 & 5 & 5 \\
Underlying 6 & 20 & 15 & 15 & 15 & 13 & 11 & 9 & 7 & 5 \\
\hline
\end{tabular}


Table 2 Option prices (in $€$ ) in the case of Contract 1 (annual premium payments of $8,400 \epsilon$; term $=5$ years)

\begin{tabular}{lrrrrrr}
\hline & \multicolumn{1}{l}{$\Pi$} & \multicolumn{1}{c}{$\Pi_{S}^{(T)}$} & \multicolumn{1}{c}{$\Pi_{K}^{(1)}$} & \multicolumn{1}{c}{$\Pi_{S}^{(1)}$} & \multicolumn{1}{c}{$\Pi_{K}^{(2)}$} & \multicolumn{1}{c}{$\Pi_{S}^{(2)}$} \\
\hline Underlying 1 & $2,080.33$ & $2,548.72$ & $2,251.86$ & $2,093.57$ & $2,490.54$ & $2,339.03$ \\
Underlying 2 & $1,172.46$ & $1,527.22$ & $1,275.18$ & $1,184.35$ & $1,467.55$ & $1,343.68$ \\
Underlying 3 & 425.04 & 629.27 & 465.46 & 433.16 & 596.97 & 526.86 \\
Underlying 4 & 956.79 & $1,360.55$ & $1,135.92$ & $1,008.44$ & $1,293.76$ & $1,166.11$ \\
Underlying 5 & 20.73 & 65.31 & 22.88 & 21.70 & 60.83 & 44.96 \\
Underlying 6 & 134.36 & 269.25 & 199.76 & 173.68 & 244.81 & 201.25 \\
\hline
\end{tabular}

Table 3 Option prices (in $€$ ) in the case of Contract 2 (annual premium payments of 4,200 $€$; term = 10 years)

\begin{tabular}{lrrrrrr}
\hline & \multicolumn{1}{c}{$\Pi$} & \multicolumn{1}{c}{$\Pi_{S}^{(T)}$} & \multicolumn{1}{c}{$\Pi_{K}^{(1)}$} & \multicolumn{1}{c}{$\Pi_{S}^{(1)}$} & \multicolumn{1}{c}{$\Pi_{K}^{(2)}$} & \multicolumn{1}{c}{$\Pi_{S}^{(2)}$} \\
\hline Underlying 1 & $1,674.17$ & $2,201.47$ & $1,837.69$ & $1,692.67$ & $2,098.98$ & $1,909.47$ \\
Underlying 2 & 833.79 & $1,196.81$ & 919.52 & 848.33 & $1,128.57$ & 991.89 \\
Underlying 3 & 207.21 & 401.87 & 229.40 & 212.89 & 360.01 & 286.56 \\
Underlying 4 & 958.07 & $1,459.37$ & $1,155.72$ & $1,031.37$ & $1,321.49$ & $1,172.16$ \\
Underlying 5 & 10.15 & 54.66 & 23.07 & 20.11 & 44.20 & 29.40 \\
Underlying 6 & 188.96 & 387.98 & 289.73 & 254.47 & 335.14 & 281.53 \\
\hline
\end{tabular}

Table 4 Option prices (in $€$ ) in the case of Contract 3 (annual premium payments of 2,100 $€$; term = 20 years)

\begin{tabular}{lrrrrrr}
\hline & \multicolumn{1}{l}{$\Pi$} & \multicolumn{1}{c}{$\Pi_{S}^{(T)}$} & \multicolumn{1}{c}{$\Pi_{K}^{(1)}$} & \multicolumn{1}{c}{$\Pi_{S}^{(1)}$} & \multicolumn{1}{c}{$\Pi_{K}^{(2)}$} & \multicolumn{1}{c}{$\Pi_{S}^{(2)}$} \\
\hline Underlying 1 & 956.98 & $1,397.60$ & $1,073.15$ & 978.12 & $1,298.77$ & $1,145.18$ \\
Underlying 2 & 380.82 & 652.95 & 427.12 & 392.76 & 599.33 & 497.36 \\
Underlying 3 & 54.44 & 164.79 & 61.06 & 57.01 & 148.05 & 99.36 \\
Underlying 4 & 663.05 & $1,063.25$ & 789.73 & 707.68 & 941.72 & 810.92 \\
Underlying 5 & 18.70 & 106.65 & 68.90 & 57.25 & 77.53 & 61.85 \\
Underlying 6 & 123.65 & 302.88 & 178.49 & 158.59 & 223.94 & 179.38 \\
\hline
\end{tabular}

Table 5 Option prices (in $€$ ) in the case of Contract 4 (annual premium payments of 1,200 $€$; term = 35 years)

\begin{tabular}{lrrrrrr}
\hline & \multicolumn{1}{l}{$\Pi$} & \multicolumn{1}{c}{$\Pi_{S}^{(T)}$} & \multicolumn{1}{c}{$\Pi_{K}^{(1)}$} & \multicolumn{1}{c}{$\Pi_{S}^{(1)}$} & \multicolumn{1}{c}{$\Pi_{K}^{(2)}$} & \multicolumn{1}{c}{$\Pi_{S}^{(2)}$} \\
\hline Underlying 1 & 381.35 & 639.46 & 432.03 & 392.19 & 571.86 & 482.64 \\
Underlying 2 & 106.43 & 250.81 & 119.74 & 110.17 & 215.79 & 161.20 \\
Underlying 3 & 4.41 & 49.06 & 5.15 & 4.83 & 37.98 & 19.97 \\
Underlying 4 & 305.88 & 521.04 & 365.24 & 327.30 & 462.26 & 388.79 \\
Underlying 5 & 31.20 & 120.62 & 87.66 & 75.70 & 93.42 & 77.98 \\
Underlying 6 & 68.81 & 175.99 & 108.44 & 91.26 & 139.60 & 110.52 \\
\hline
\end{tabular}

option is exercised annually, and $\Pi^{(1)}\left(\right.$ or $\left.\Pi^{(2)}\right)$ represents the price if the paid-up option is exercised once without (or with) entering a new contract. In the latter case, we distinguish the option price $\Pi_{K}^{(\cdot)}$ (the investor discovers the optimal date to stop premium payments by mere chance) from $\Pi_{S}^{(\cdot)}$ (value under the optimal admissible stopping date; calculated applying the exercise value threshold method (EVTM)). 
Tables 2-5 show that the options are of substantial value. In absolute terms, the highest value $(2,548.72 €)$ occurs with Contract 1 and Underlying 1, where the investor exercises the paid-up option annually. Here, the value of the option is more than $6 \%$ of the sum of the premiums paid.

The value is the highest when the client chooses to exercise the paid-up option annually. For example, Contract 4, Underlying 3 shows that the difference in relative terms can be very large between the situation where the investor chooses to exercise the paid-up option annually and the situation where all premiums are paid until maturity $\left(\Pi_{S}^{(T)}=49,06 € ; \Pi=4,41 €\right)$.

Tables 2-5 also illustrate that there is always a substantial additional risk for the seller of subsidized pension products if the client chooses to stop premium payment (at least once) at some time during the life of the contract. Of course, the value of the pure money-back guarantee $(\Pi)$ is always increased by the paid-up option.

Given a constant volatility of the underlying, the option prices will ceteris paribus decline as the term of the contract increases. On the other hand, less volatility for a given contract duration will c.p. lessen the value of the options. However, if there is a volatility structure that declines as the contract nears its term (Underlyings 4-6), the value of the option is still substantial—especially if the client may make the contract paid-up. It is particularly interesting that the value of the pure money-back guarantee ( $\Pi$ ) decreases quickly when we have a decreasing volatility structure, whereas the additional value resulting from the paid-up option $\left(\Pi_{a}^{(b)}-\Pi, a \in\{S, K\}, b \in\{1,2, T\}\right)$ decreases slower or can even increase. For example, for Contract 1 , the value $\Pi$ decreases by $54 \%$ when Underlying 1 is replaced by Underlying 4 . However, $\Pi_{S}^{(T)}-\Pi$ decreases by merely $14 \%$ and $\Pi_{K}^{(1)}-\Pi$ increases by more than $4 \%$. Thus, shifting the client's assets from stocks to bonds (i.e., reducing volatility) as maturity approaches is appropriate for reducing the risk resulting from the pure money-back guarantee, but much less effective in reducing the risk resulting from the paid-up option.

As illustrated in Section 4.3, the maximum price of the option per contract is obtained if the client exercises the paid-up option once and by mere chance chooses the optimal exercise date $\tau$ (the option price denoted by $\Pi_{K}^{(1)}$ in Tables $2-5$ ). ${ }^{9}$ Of course, realistically, not very many, if any, clients will find the optimal exercise date by mere chance. Also, most likely, only a very few clients will use the exercise value threshold method (EVTM), as described in Section 4.3, to approximate an optimal stopping strategy (the option price denoted by $\Pi_{K}^{(2)}$ in Tables 2-5). Clients may have many reasons for stopping payment of premiums, reasons that have very little to do with any sort of optimal stopping decisions. The client may be in financial straits or simply might want to spend the money on something else. As these governmentsubsidized pension products have been available for only three years, there is as yet no empirical information regarding whether observed and optimal stopping decisions coincide. However, even if empirical stopping decisions information were available, we are not convinced that it would actually be a good estimator for the future. As time goes by, and consumers become more familiar with these products, it can be assumed that clients will have more and better information to help them make optimal stopping

\footnotetext{
9 This holds true in most cases because most providers do not allow their clients to use the paid-up option more than once per contract.
} 
decisions. One can even imagine specialist consulting agencies that will advise clients on the optimal point at which to stop paying premiums into the contract. Although not all clients, even in the future, will be so well-informed or well-advised, we still strongly believe that sellers of subsidized pension products should pay close attention to our findings because it is entirely possible that after a market drop, disappointed clients will typically make a contract paid-up at a point when the asset value of the contract is particularly low, which is not so different from an optimal stopping decision.

Therefore, sellers of government-subsidized pension products must make assumptions about client behavior concerning the exercise of the option in order to adequately price the per-contract money-back-guarantee. Assuming clients to follow some kind of optimal strategy could be one approach. Including the concept of exercise probabilities into the standard risk-neutral evaluation methodology, as proposed by Dillmann and Russ [2003], could be an alternative approach that allows considering non-financial aspects of the exercise behavior.

However, from the seller's point of view, it is not sufficient to price the contract and merely receive the value of the put option from the customer. Instead, the received option premium has to be used to finance adequate risk management measures. In an arbitrage-free market without transaction costs, the value of the put option is exactly the price of any risk management measure ensuring the given guarantee, including, for example, a hedging strategy, an insurance contract, contingent capital, or an increment of the company's equity capital.

\section{Summary}

In Germany, newly introduced government-subsidized pension products are required by law to contain a promise by the seller of a "money-back guarantee" at the end of the term. Hence, at minimum, the premiums paid into the contract have to be available at the end of the accumulation phase. The client is also given the right to stop paying premiums at any time (paid-up option), in which case, the amount of all premiums paid must also be guaranteed by the seller at maturity, regardless of when the client stopped paying the premiums.

Previous analysis focused on the pure money-back guarantee, i.e., the asset value guarantee if all premiums are paid as scheduled (cf. Gründl et al. [2004]), and it was found that this option can have a significant value. Our analysis confirms this finding. Furthermore, we focused on the paid-up option, i.e., the asset value guarantee if the client stops paying premiums at some time during the term of the contract, and found that this additional option, which is required by law, has a significant additional value.

Many product providers try to reduce their risk by reducing volatility as time to maturity decreases. Our analysis shows that this is a suitable strategy for reducing the risk resulting from the pure money-back guarantee, but much less effective in reducing the risk resulting from the paid-up option.

In mutual funds, the embedded options are often given away for free; hence, the contracts generally are not priced appropriately, because the seller of such subsidized pension products needs to receive the value of the put option from the clients in order to finance adequate risk management measures. 
We expect knowing and acting upon (in the pricing context) the fair value of embedded options will become increasingly important. The recent case of Equitable Life, a British life insurer that got in financial trouble because of improperly hedged embedded options, demonstrates that it is necessary to quantify and manage the risk associated with such options.

\section{Appendix A: Calculation of the value $\Pi^{(1)}(K)$ of the option assuming that the investor applies some given strategy $K=K_{1} \times \cdots \times K_{T-1} \subseteq R^{T-1}$}

1. Generate random paths $S_{1}^{i}, \ldots, S_{T}^{i}$ of the underlying for $i=1, \ldots$, sim using Equation (12). ${ }^{10}$

2. Let $K_{T}=R_{+}{ }^{11}$

3. Calculate $\tau^{(i)}(i=1, \ldots, \operatorname{sim})$ as follows:

a. Let $i=1$.

b. Let $t=1$.

c. Calculate the value of the assets in the contract $V_{t}^{-}=\sum_{v=0}^{t-1} P \frac{S_{t}^{i}}{S_{v}^{i}}$ using the $i$-th random path of the underlying generated in Step 1.

d. If $V_{t}^{-} \notin K_{t}$, increase $t$ by 1 and go to Step 3.c.

e. Let $\tau^{(i)}=t$.

f. If $i<\operatorname{sim}$, increase $i$ by 1 and go to Step 3.b.

4. For each $i$, calculate $V_{T}^{* \tau^{(i)}}=\sum_{\nu=0}^{\tau^{(i)}-1} P \frac{S_{T t}^{i}}{S_{v}^{i}}$ using $\tau^{(i)}$ calculated in Step 3.

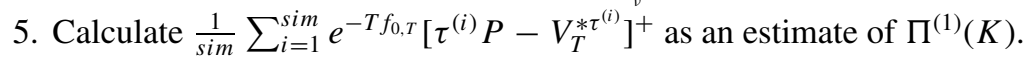

Appendix B: Calculation of the value $\Pi_{S}^{(1)}$ of the option by approximating an optimal strategy $K=\left(0 ; k_{1}\right] \times \cdots \times\left(0 ; k_{T-1}\right]$

1. Generate random paths $S_{1}^{i}, \ldots, S_{T}^{i}$ of the underlying for $i=1, \ldots$, sim.

2. Approximate the optimal strategy $k_{1}, \ldots, k_{T-1}$ as follows:

a. Let $t=T-1$.

b. For $k \in[0,2]$ in steps of 0.01 calculate ${ }^{12} \Pi^{(1)}\left(0, \ldots, 0, k, k_{t+1}, \ldots, k_{T-1}\right)$ using the algorithm described in Appendix A.

c. Choose $k_{t}$ such that $\Pi^{(1)}\left(0, \ldots, 0, k_{t}, k_{t+1}, \ldots, k_{T-1}\right)=\max _{k} \Pi^{(1)}\left(0, \ldots, 0, k, k_{t+1}, \ldots, k_{T-1}\right)$.

d. If $t>1$, decrease $t$ by 1 and go to Step 2.b.

3. Calculate $\Pi^{(1)}\left(k_{1}, \ldots, k_{T-1}\right)$ using the algorithm described in Appendix $\mathrm{A}$ as an estimate of $\Pi_{S}^{(1)}$.

\footnotetext{
$\overline{10}$ If this algorithm is used within the algorithm given in Appendix B, omit Step 1.

${ }^{11}$ This ensures that payments are stopped at the latest at $\mathrm{T}$.

${ }^{12}$ Interval and step length were chosen by experience.

Springer
} 


\section{Appendix C: Calculation of the value $\Pi^{(2)}(\tilde{K})$ of the option by applying} some given strategy $\tilde{K}=\tilde{K}_{1} \times \cdots \times \tilde{K}_{T-1} \subseteq R^{T-1}$ assuming that the investor enters a second contract when making the first contract paid-up

1. Generate random paths $S_{1}^{i}, \ldots, S_{T}^{i}$ of the underlying for $i=1, \ldots$, sim using Equation (12). ${ }^{13}$

2. Let $\tilde{K}_{T}=R_{+}{ }^{14}$

3. Calculate $\tau^{(i)}(i=1, \ldots, \operatorname{sim})$ as follows:

a. Let $i=1$.

b. Let $t=1$.

c. Calculate the value of the assets in the contract $V_{t}^{-}=\sum_{v=0}^{t-1} P \frac{S_{t}^{i}}{S_{v}^{i}}$ using the $i$-th random path of the underlying generated in Step 1.

d. If $V_{t}^{-} \notin \tilde{K}_{t}$, increase $t$ by 1 and go to Step 3.c.

e. Let $\tau^{(i)}=t$.

f. If $i<\operatorname{sim}$, increase $i$ by 1 and go to Step 3.b.

4. For each $i$, calculate $V_{T}^{* \tau^{(i)}}=\sum_{\nu=0}^{\tau^{(i)}-1} P \frac{S_{T t}^{i}}{S_{v}^{i}}$ and $W_{T}^{* \tau^{(i)}}=\sum_{\nu=\tau^{(i)}}^{T-1} P \frac{S_{T t}^{i}}{S_{\nu}^{i}} \operatorname{using} \tau^{(i)}$ calculated in Step 3.

5. Calculate $\frac{1}{\operatorname{sim}} \sum_{i=1}^{\operatorname{sim}} e^{-T f_{0, T}}\left(\left[\tau^{(i)} P-V_{T}^{* \tau^{(i)}}\right]^{+}+\left[\left(T-\tau^{(i)}\right) P-W_{T}^{* \tau^{(i)}}\right]^{+}\right)$as an estimate of $\Pi^{(2)}(\tilde{K})$.

\section{Appendix D: Calculation of the value $\Pi_{S}^{(2)}$ of the option by approximating an} optimal strategy $\tilde{\boldsymbol{K}}=\left(\mathbf{0} ; \tilde{\boldsymbol{k}}_{1}\right\rfloor \times \cdots \times\left(\mathbf{0} ; \tilde{\boldsymbol{k}}_{T-1}\right\rfloor$

Note: In the case where a second contract is entered into after making the first contract paid-up, it is never optimal to not exercise the paid-up option at all. Thus, if the option has not been exercised until time $T-1$, it is optimal to exercise at time $T-1$. For instance, assume the option has not been exercised until time $T-1$. If the client decides not to exercise at time $T-1$, the payoff will be $L_{T}=V_{T}+\left[T P-V_{T}\right]^{+}$. If the client exercises at time $T-1$, the payoff is given by $\tilde{L}_{T}^{* T-1}=\max \left\{V_{T}^{* T-1} ;(T-1) P\right\}+\max \left\{W_{T}^{* T-1} ; P\right\}$ (using the terminology from Section 4.4). Some easy calculation shows that $\tilde{L}_{T}^{* T-1}>L_{T}$. Thus, we let $\tilde{k}_{T-1}=\infty$ in Step 2 of the following algorithm.

1. Generate random paths $S_{1}^{i}, \ldots, S_{T}^{i}$ of the underlying for $i=1, \ldots$, sim.

2. Let $\tilde{k}_{T-1}=\infty$.

3. Approximate the optimal strategy $\tilde{k}_{1}, \ldots, \tilde{k}_{T-2}$ as follows:

a. Let $t=T-2$.

b. For $k \in[0,8]$ in steps of 0.01 calculate $^{15} \Pi^{(2)}\left(0, K, 0, k, \tilde{k}_{t+1}, K, \tilde{k}_{T-1}\right)$ Using the algorithm described in Appendix C.

\footnotetext{
${ }^{13}$ If this algorithm is used within the algorithm given in Appendix D, omit Step 1.

14 This ensures that payments are stopped at the latest at T.

15 Interval and step length were chosen by experience.
} 
c. Choose $\tilde{k}_{t}$ such that $\Pi^{(2)}\left(0, \ldots, 0, \tilde{k}_{t}, \tilde{k}_{t+1}, \ldots, \tilde{k}_{T-1}\right)=\max _{k} \Pi^{(2)}\left(0, \ldots, 0, k, \tilde{k}_{t+1}, \ldots, \tilde{k}_{T-1}\right)$.

d. If $t>1$, decrease $t$ by 1 and go to Step 2.b.

4. Calculate $\Pi^{(2)}\left(\tilde{k}_{1}, \ldots, \tilde{k}_{T-1}\right)$ using the algorithm described in Appendix $\mathrm{C}$ as an estimate of $\Pi_{S}^{(2)}$.

\section{References}

ANDERSON, L. [1999]: "A Simple Approach to the Pricing of Bermudan Swaptions in the Multi-factor Labor Market Model," Journal of Computational Finance, 3, 5-32.

DILLMANN, T. and RUSS, J. [2003]: "Implicit Options in Life Insurance Contracts—From Option Pricing to the Price of the Option," Working Paper, University of Ulm.

DOUADY, R. [2002]: "Bermudan Option Pricing with Monte Carlo Methods," in Quantitative Analysis in Financial Markets, Volume III. World Scientific Publishing Co. pp. 314-328.

GRÜNDL, H., NIETERT, B., and SCHMEISER, H. [2004]: "Zur Zusage der nominalen Kapitalerhaltung bei investmentfondsorientierten Riester-Produkten: Einige Überlegungen aus finanzierungstheoretischer Sicht," Zeitschrift für Betriebswirtschaft, 74, 119-137.

HARRISON, J.M. and KREPS, D.M. [1979]: "Martingales and Arbitrage in Multiperiod Securities Markets," Journal of Economic Theory, 20, 381-408.

HARRISON, J.M. and PLISKA, S.R. [1981]: "Martingales and Stochastic Integrals in the Theory of Continuous Trading," Stochastic Processes and Their Applications, 11, 215-260.

HULL, J.C. [2003]: Options, Futures, and Other Derivatives, 5th ed. Englewood Cliffs, NJ: Prentice-Hall, Inc.

LACHANCE, E.-M. and MITCHELL, O.S. [2003]: "Understanding Individual Account Guarantees," in The Pension Challenge: Risk Transfers and Retirement Income Security. Pension Research Council. O.S. Mitchell and K. Smetters (Eds.), Oxford, UK: Oxford University Press, pp. 159-186.

RUSS, J. [1999]: "Die aktienindexgebundene Lebensversicherung mit garantierter Mindestverzinsung in Deutschland," IFA-Verlag, Ulm. 